\title{
Efficient Synthesis of 1-Thiomansonones with Anti-MRSA Activity
}

\author{
Seong-Hyuk Park ${ }^{\mathrm{\triangleright} \diamond}$ \\ Sooyoung Park ${ }^{\mathrm{a} \diamond}$ \\ Chang-Yong Lee ${ }^{a}$ \\ Young-Ger Suh \\ Dongyun Shin*a \\ a College of Pharmacy, Gachon University, 191 Hambakmoe-ro, \\ Yeonsu-gu, Incheon 21936, South Korea \\ dyshin@gachon.ac.kr \\ ${ }^{b}$ College of Pharmacy, CHA University, 120 Haeryong-ro, \\ Pocheon-si, Gyeonggi-do, Korea \\ $\diamond$ S.-H. Park and S. Park contributed equally to this work.
}

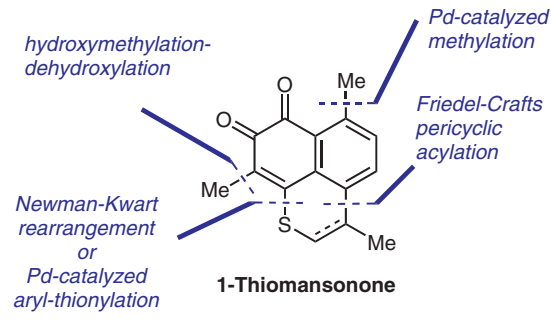

\begin{tabular}{lc}
\multicolumn{2}{c}{ Anti-MRSA Activity } \\
\hline Compound & MIC $_{50}(\mu \mathrm{g} / \mathrm{ml})$ \\
\hline Mansonone I & 8 \\
1-Thiomansonone I & 16 \\
1-Sulfonmansonone I & $>32$ \\
Mansonone F & 2 \\
1-Thiomansonone F & 8 \\
\hline
\end{tabular}

Received: 06.11.2017

Accepted after revision: 12.12.2017

Published online: 29.01 .2018

DOI: 10.1055/s-0036-1591894; Art ID: st-2017-u0821-

Abstract In this study, we developed an efficient and general synthetic strategy for thiaphenalene, a sulfur-containing polyaromatic heterocycle, and applied for the synthesis of 1-thio derivatives of mansonone I and $\mathrm{F}$, natural 1-oxaphenalenic orthoquinones. The pivotal steps for the construction of thiophenalene skeleton include formation of arylsulfide by Newman-Kwart rearrangement of thiocarbamate or palladiumcatalyzed cross-coupling, and pericyclic ring closure. Three bioisosterically modified orthoquinones were synthesized and were evaluated for anti-MRSA activity.

Key words Newman-Kwart, mansonones, palladium, orthoquinones, thiaphenalene, MRSA

Methicillin-resistant Staphylococcus aureus (MRSA) is one of the most important pathogenic bacteria. ${ }^{1,2}$ Rapid acquisition of multidrug resistance to various antibiotics and severe nosocomial infections caused by MRSA have become major problems in the clinic. ${ }^{2}$ Despite recent introduction of several new classes of antibiotics, including linezolid, quinupristin/dalfopristin, and new glycopeptide antibiotics, drug resistance, potency, and toxicity remain unsolved problems. ${ }^{1}$ Therefore, discovery of novel antiMRSA agents persists as a focus of medicinal chemistry efforts. $^{3-5}$

One of the continuing research interests in our laboratory is the synthesis and evaluation of structurally novel small molecules with potent anti-MRSA activity, which could overcome multidrug resistance. We previously reported the isolation and synthesis of mansonone $F$, found in root bark of Ulmus davidiana, a Korean medicinal plant (Figure 1). ${ }^{6,7}$ Mansonone $\mathrm{F}$ is a phytoalexin ${ }^{8}$ and structurally belongs to naturally occurring sesquiterpenoid orthoquinones, with many congeners reported from different plants. ${ }^{9-11}$ Mansonones have a unique oxaphenalene skele- ton and, due to orthoquinone moiety and high degree of conjugation, exhibit distinct colors in solution, e.g., deep purple color of $\mathrm{CHCl}_{3}$ solution of mansonone $\mathrm{F}$. Most importantly, mansonones show potent anti-MRSA activity comparable with vancomycin, a drug of choice for treating MRSA infections. ${ }^{12}$<smiles></smiles><smiles></smiles>
Mansonone I<smiles></smiles>
Mansonone E

Figure 1 Structures of natural mansonone congeners

Systematic structure-activity relationship study of mansonone $\mathrm{F}$ indicated that any change of the tricyclic oxaphenalene skeleton and the orthoquinone moiety markedly reduced anti-MRSA activity, implying that these features are essential pharmacophores for antibacterial activity. Maintaining the tricyclic orthoquinone core structure, subsequent SAR study showed that 1) loss of C1-C2 double bond slightly reduced the activity, and 2) any polar groups or electron-withdrawing ones at C3 also reduced or eliminated the potency, while saturated alkyl chains have little effect on activities. Most of the C6, C9 analogues were less potent than mansonone $\mathrm{F}$ regardless of substituents, while the steric effect was proven to be more important than the electronic effect. Unexpectedly, the 6-butylmansonone presented exceptionally powerful anti-MRSA and antibacterial effect for the Gram-positive pathogens. ${ }^{13,14}$

To discover new and more potent anti-MRSA compounds we extended the SAR analysis to 1-thiomansonone derivatives. We hypothesized that as orthoquinone moiety and oxaphenalene core are crucial for antimicrobial activity and as the structure is fully conjugated, substitution of 
oxygen in oxaphenalene with other atoms such as sulfur would alter electronic characteristics, and therefore biological activity of the compounds. Sulfur is one of the classic oxygen bioisosteres, and bioisosteric displacement is frequently used in medicinal chemistry. ${ }^{15-17}$ However, introduction of the sulfhydryl group on the benzene ring, directly or by functional group interconversion, has proven synthetically challenging. In this communication, we report efficient syntheses of 1-thio derivatives (1, 2, and 3) of mansonone and evaluation of their anti-MRSA activities (Figure 2).

Synthetic strategy and retrosynthetic plan for the synthesis of 1-thio mansonones are briefly outlined in Figure 3 and Scheme 1.
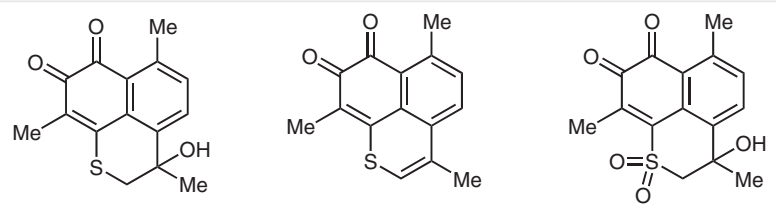

1-Thiomansonone I (1)

1-Thiomansonone F (2)

1-Sulfonmansonone I (3)

Figure 2 Structures of 1 -thio and 1-sulfonmansonone $I$ and $F$

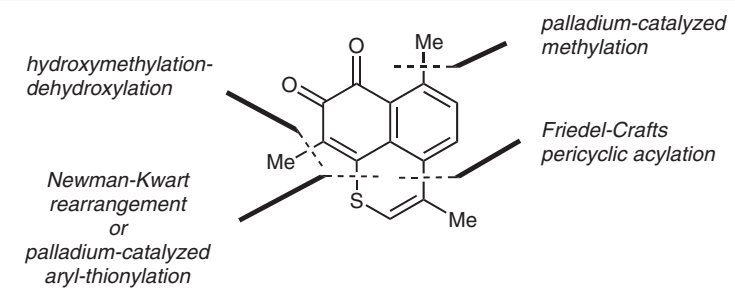

Figure 3 Synthetic strategy<smiles></smiles>

1-Thiomansonone $\mathrm{F}$<smiles>Cc1ccc2c(C)cccc2c1O</smiles>

6<smiles>Cc1ccc2c(C)ccc3c2c1C(=O)CS3</smiles>

4<smiles>Oc1cccc2c(O)cccc12</smiles>

Scheme 1 Retrosynthesis

1-Thiomansonone can be synthesized from tricyclic ketone 4, whereas the tricyclic thiophenalene skeleton can be constructed by Friedel-Crafts peri ring closure of acid $\mathbf{5}$. The key reaction of this synthesis is the creation of the arylsulfide bond. Formation of the carbon-sulfur bond could be achieved by Newman-Kwart rearrangement ${ }^{18}$ of an aryl thiocarbamate or by palladium-catalyzed cross-coupling of aryl 0 -triflate, starting from naphthol $\mathbf{6}$ and an appropriate thiol. ${ }^{19}$ 6- and 9-Methyl groups could be introduced by palladium-catalyzed methylation and sequential orthohydroxymethylation-benzylic dihydroxylation from 1,5dihydroxynaphthalene (7), respectively. 2,5-Dimethylnaphth-1-ol (6) was prepared by modifying our previously established procedure (Scheme 2 ).

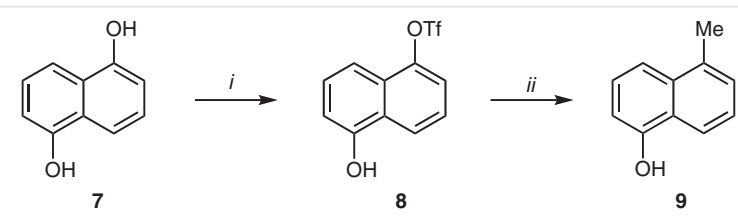<smiles>CC#CCCCCC</smiles><smiles>Cc1ccc2c(C)cccc2c1O</smiles>

6

Scheme 2 Synthesis of naphthol 6. Reagents and conditions: i. PhN(Tf $)_{2}$, DMAP, Et ${ }_{3} \mathrm{~N}, \mathrm{rt}, 45 \%$ ii. $\mathrm{MeB}(\mathrm{OH})_{2}$, $\mathrm{Pd}(\mathrm{dppf}) \mathrm{Cl}_{2}, \mathrm{~K}_{2} \mathrm{CO}_{3}, \mathrm{THF} / \mathrm{H}_{2} \mathrm{O}(10: 1)$, reflux, $78 \%$ iii. a. $\mathrm{PhB}(\mathrm{OH})_{2},\left(\mathrm{CH}_{2} \mathrm{O}\right)_{n}, \mathrm{CH}_{3} \mathrm{CH}_{2} \mathrm{CO}_{2} \mathrm{H}$, benzene, reflux b. $\mathrm{H}_{2}, 10 \% \mathrm{Pd} / \mathrm{C}$, EtOH/THF, c-HCl, rt, $84 \%$ for 2 steps.

Commercially available 1,5-dihydroxynaphthalene (7) was treated with $N$-phenylbis(trifluoromethanesulfonimide) ( $\mathrm{PhNTf}_{2}$ ), DMAP, and $\mathrm{Et}_{3} \mathrm{~N}$ in $\mathrm{CH}_{2} \mathrm{Cl}_{2}$ to give monotrifluoromethanesulfonylated naphthol $(\mathbf{8}),{ }^{20}$ which was subjected to Suzuki reaction using [1,1'-bis(diphenylphosphino)ferrocene] dichloropalladium(II) $\left[\mathrm{Pd}(\mathrm{dppf}) \mathrm{Cl}_{2}\right]$ as a catalyst and methylboronic acid as a methyl donor under THF reflux to obtain 5-methyl-1-naphthol (9) in $78 \%$ yield. ${ }^{21}$ ortho-Hydroxymethylation of naphthol $\mathbf{7}$ in the presence of phenylboronic acid, paraformaldehyde, and propionic acid in benzene yielded boronic ester $\mathbf{1 0},{ }^{22}$ which was readily converted into 2,5-dimethylnaphthol in excellent yield by hydrogenolysis without any purification steps. The reaction was performed using crude boronic ester under the following conditions: $\mathrm{H}_{2}$ (balloon), $10 \% \mathrm{Pd} / \mathrm{C}$, $\mathrm{EtOH} / \mathrm{THF}$, and catalytic amount of $c-\mathrm{HCl}$ at room temperature.

Methods for introducing an alkylthio group at an aryl ring include substitution of aryl halides or diazonium salts with sulfur nucleophiles, intramolecular rearrangement of arylthiocarbamates, known as Newman-Kwart rearrangement, and transition-metal-catalyzed coupling of arylhalides or aryltriflates with alkyl or aryl mercaptanes developed by the Buchwald group. Our first attempt to synthesize compound $\mathbf{5}$ used thermal Newman-Kwart rearrangement, as shown in Scheme 3. 


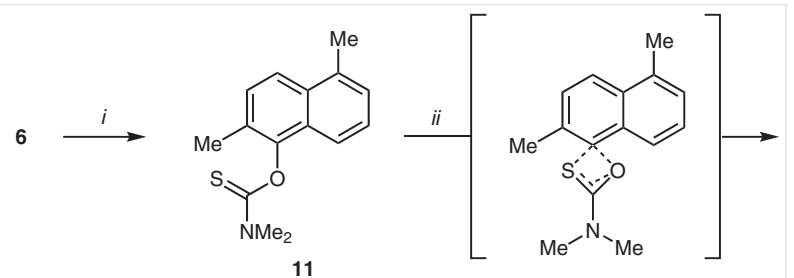<smiles>CC(=O)CSc1c(C)ccc2c(SCC(=O)O)c(C)ccc12</smiles>

Scheme 3 Newman-Kwart rearrangement approach for thioether synthesis. Reagents and conditions: i. N,N-dimethylthiocarbamoyl chloride, pyridine, rt, $75 \%$ ii. $\mathrm{N}, \mathrm{N}$-dimethylacetamide, reflux, $71 \%$ iii. $4 \mathrm{~N} \mathrm{KOH}$, $\mathrm{MeOH}$, reflux, $85 \%$ iv. iodoacetic acid, $\mathrm{NaH}, \mathrm{DMF}, \mathrm{rt}, 76 \%$.

$\mathrm{N}, \mathrm{N}$-Dimethylthiocarbamoyl group was introduced to naphthol 6, followed by thermal Newman-Kwart rearrangement under $\mathrm{N}, \mathrm{N}$-dimethylacetamide reflux to obtain the desired carbamothioate $\mathbf{1 2}$ in good yield. ${ }^{23}$ Carbamoyl moiety in $\mathbf{1 2}$ was readily removed with $\mathrm{KOH}$ treatment to yield naphthyl thiol 13, which was alkylated with iodoacetic acid to generate $\mathbf{5}$. Additionally, we designed an alternate synthesis route, a transition-metalcatalyzed thioalkylation between aryl halides or triflate and a thiol donor (Scheme 4).<smiles>Cc1ccc(C)c2c(C)cccc12</smiles>

14

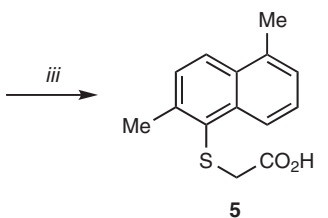

Scheme 4 Palladium-catalyzed cross-coupling approach to thioether synthesis. Reagents and conditions: $i$. PhN(Tf $)_{2}$, DMAP, $\mathrm{Et}_{3} \mathrm{~N}, \mathrm{rt}, 95 \%$ ii. $\mathrm{HSCH}_{2} \mathrm{CO}_{2} \mathrm{CH}_{3}, \mathrm{Pd}_{2}(\mathrm{dba})_{2}$, dppf, $\mathrm{NaOt}$ - $\mathrm{Bu}$, toluene, reflux, $43 \%$ iii. $\mathrm{LiOH} \cdot \mathrm{H}_{2} \mathrm{O}, \mathrm{THF} / \mathrm{H}_{2} \mathrm{O}(3: 1)$, rt, $92 \%$

Naphthyl-O-triflate $\mathbf{1 4}^{20}$ was prepared by treating naphthol $\mathbf{6}$ with $\mathrm{PhN}(\mathrm{Tf})_{2}$ and used for the cross-coupling. Methyl 2-mercaptoacetate was successfully introduced to the naphthyl ring using tris(dibenzylideneacetone)-dipalladium catalyst and 1,1'-bis(diphenylphosphino)-ferrocene ligand, sodium tert-butoxide as base, in toluene, to afford methyl 2-(1,6-dimethylnaphthalen-5-ylthio)acetate (15) in $43 \%$ yield. $^{24}$ The ester was hydrolyzed to give acid $\mathbf{5}$.
Tricyclic ketone $\mathbf{4}$ was synthesized using an intramolecular pericyclic Friedel-Crafts reaction. Generation of acid chloride from acid $\mathbf{1 4}$ using oxalyl chloride, followed by aluminum chloride treatment resulted in the desired ketone 4 in 90\% yield in two steps. Methyl substituent at C3 was introduced by Grignard reaction (MeMgI, $\mathrm{Et}_{2} \mathrm{O}$ ). Sequential nitration at $\mathrm{C} 7$ by copper nitrate and reduction to aniline followed by oxidation with Fremy's salt, delivered 1-thiomansonone I (1). ${ }^{25}$ Thiomansonone I (1) was dehydrated to 1-thiomansonone $\mathrm{F}(\mathbf{2})$ according to a previously described procedure for the synthesis of mansonone $\mathrm{F}$ from mansonone I, as shown in Scheme 5. Sulfone derivative 3 of mansonone I was synthesized by sulfide oxidation of $\mathbf{1}$ with mCPBA.

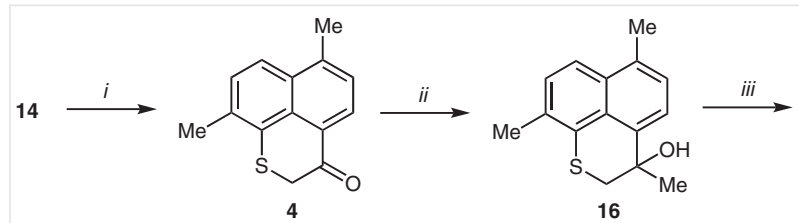

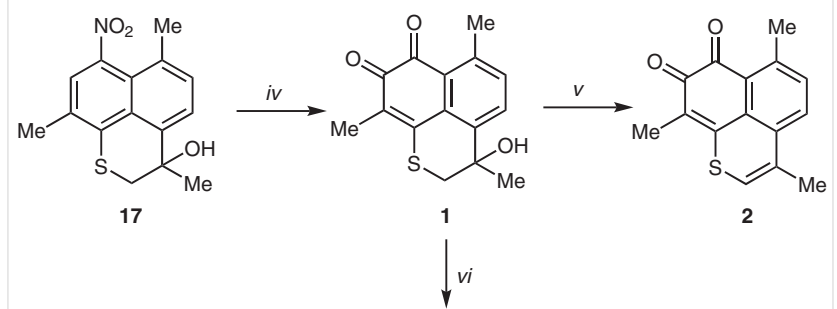<smiles></smiles>

Scheme 5 Synthesis of thiomansonones. Reagents and conditions: i. $(\mathrm{COCl})_{2}$, benzene, reflux; $\mathrm{AlCl}_{3}, \mathrm{CH}_{2} \mathrm{Cl}_{2}, 0^{\circ} \mathrm{C}, 90 \%$ ii. $\mathrm{CH}_{3} \mathrm{Mgl}$, Et $\mathrm{Et}_{2} \mathrm{O}$, rt, $81 \%$ iii. $\mathrm{Cu}\left(\mathrm{NO}_{3}\right)_{2} \cdot \mathrm{xH}_{2} \mathrm{O}, \mathrm{Ac}_{2} \mathrm{O}, \mathrm{rt}, 83 \%$ iv. $\mathrm{H}_{2}, \mathrm{Pd} / \mathrm{C}, \mathrm{MeOH}$; Fremy's salt, $0.06 \mathrm{M} \mathrm{NaH}_{2} \mathrm{PO}_{4}$, acetone, $47 \%$ for two steps v. c- $\mathrm{H}_{2} \mathrm{SO}_{4} / \mathrm{EtOH}$ (1:20), reflux, $35 \%$ vi. $\mathrm{mCPBA}, \mathrm{CH}_{2} \mathrm{Cl}_{2}, 0{ }^{\circ} \mathrm{C}, 67 \%$.

As depicted in Table 1, antimicrobial activities of the synthesized analogues were determined in vitro against MRSA strains. Vancomycin, mansonone I, and mansonone F were used as control compounds.

As previously reported, mansonone $\mathrm{F}\left(\mathrm{MIC}_{50}=2 \mu \mathrm{g} / \mathrm{mL}\right)$ shows anti-MRSA activity comparable to vancomycin $\left(\mathrm{MIC}_{50}=1 \mu \mathrm{g} / \mathrm{mL}\right)$, whereas mansonone I is slightly less potent $\left(\mathrm{MIC}_{50}=8 \mu \mathrm{g} / \mathrm{mL}\right)$. Thiomansonone I (1) displayed slightly lower activity $\left(\mathrm{MIC}_{50}=16 \mu \mathrm{g} / \mathrm{mL}\right)$ compared to mansonone I, whereas 1-sulfonmansonone I (3) showed no notable anti-MRSA activity $\left(\mathrm{MIC}_{50}>32 \mu \mathrm{g} / \mathrm{mL}\right.$ ) in the concentration range tested. Dehydrated analogue, 1-thiomanonone $F(2)$ showed mild anti-MRSA activity, but did not enhance the antibacterial activity compared with mansonone F. In designing this experiment, we expected that, 
Table 1 Anti-MRSA Activity

\begin{tabular}{llcc}
\hline Entry & Compound & $\mathrm{MIC}_{50}(\mu \mathrm{g} / \mathrm{mL})$ & $\mathrm{MIC}_{90}(\mu \mathrm{g} / \mathrm{mL})$ \\
\hline 1 & vancomycin & 1 & 2 \\
2 & mansonone I & 8 & 16 \\
3 & 1-thiomansonone I (1) & 16 & 16 \\
4 & 1-sulfonmansonone I (3) & $>32$ & $>32$ \\
5 & mansonone F & 2 & 2 \\
6 & 1-thiomansonone F (2) & 8 & 16 \\
\hline
\end{tabular}

based on the differences in electronegativity and reactivity, substitution of 1-oxygen with sulfur or sulfone moiety would make a significant influence on compound antiMRSA activity. However, 1-thio and 1-sulfonmansonones displayed reduced anti-MRSA activity.

In conclusion, we designed new 1-thio- and 1-sulfonmansonones I and $\mathrm{F}$ based on previous SAR studies and carried out total synthesis of three sulfur-substituted analogues of mansonone $\mathrm{F}$. The two methyl substituents were introduced using Stille reaction and sequential hydroxymethylation-hydrogenolysis, respectively. The carbonsulfur bond was formed through Newman-Kwart rearrangement or palladium-catalyzed thioalkylation, whereas intramolecular Friedel-Crafts cyclization completed the tricyclic ring system. In summary, three sulfur-containing mansonone analogues were successfully synthesized and evaluated for anti-MRSA activity. The compounds did not show enhanced activity; however, our results provide valuable information for future mechanistic and SAR studies.

\section{Funding Information}

This work was supported by the National Research Foundation of Korea (NRF-2015R1D1A1A01056620 and NRF-2014M3C1A3054139) and partly the Korea Health Technology R\&D Project through the Korea Health Industry Development Institute (KHIDI), Ministry of Health and Welfare (grant No. HI14C1135).

\section{Supporting Information}

Supporting information for this article is available online at https://doi.org/10.1055/s-0036-1591894.

\section{References and Notes}

(1) Holland, T. L.; Arnold, C.; Fowler, V. G. Jr. JAMA 2014, 312, 1330.

(2) Ippolito, G.; Leone, S.; Lauria, F. N.; Nicastri, E.; Wenzel, R. P. Int. J. Infect. Dis. 2010, 14, S7; Suppl. 4.

(3) Mohammad, H.; Mayhoub, A. S.; Ghafoor, A.; Soofi, M.; Alajlouni, R. A.; Cushman, M.; Seleem, M. N. J. Med. Chem. 2014, 57, 1609.
(4) Koh, J.-J.; Lin, S.; Aung, T. T.; Lim, F.; Zou, H.; Bai, Y.; Li, J.; Lin, H.; Pang, L. M.; Koh, W. L.; Salleh, S. M.; Lakshminarayanan, R.; Zhou, L.; Qiu, S.; Pervushin, K.; Verma, C.; Tan, D. T. H.; Cao, D.; Liu, S.; Beuerman, R. W. J. Med. Chem. 2014, 58, 739.

(5) Pasberg-Gauhl, C. Drug Discovery Today: Technol. 2014, 11, 109.

(6) Shin, D.; Kim, H.-S.; Min, K.-H.; Hyun, S.-S.; Kim, S.-A.; Huh, H.; Choi, E.-C.; Choi, Y. H.; Kim, J.; Choi, S.-H.; Kim, W.-B.; Suh, Y.-G. Chem. Pharm. Bull. 2000, 48, 1805.

(7) Suh, Y.-G.; Shin, D.; Min, K.-H.; Hyun, S.-S.; Jung, J.-K.; Seo, S.-Y. Chem. Commun. 2000, 1203.

(8) Banerjee, A. K.; Laya, M. S.; Poon, P. S. In Studies in Natural Products Chemistry; Atta-ur-Rahmann, Ed.; Elsevier: Amsterdam, 2006, Part M, Vol. 33193.

(9) Kim, J.-P.; Kim, W.-G.; Koshino, H.; Jung, J.; Yoo, I.-D. Phytochemistry 1996, 43, 425.

(10) Overeem, J. C.; Elgersma, D. M. Phytochemistry 1970, 9, 1949.

(11) Bettòlo, G. B. M.; Casinovi, C. G.; Galeffi, C. Tetrahedron Lett. 1965, 6, 4857.

(12) Rubinstein, E.; Keynan, Y. Front. Public Health 2014, 2, 217.

(13) Suh, Y. G.; Kim, S. N.; Shin, D.; Hyun, S. S.; Lee, D. S.; Min, K. H.; Han, S. M.; Li, F.; Choi, E. C.; Choi, S. H. Bioorg. Med. Chem. Lett. 2006, 16, 142 .

(14) Shin, D.; Kim, S. N.; Chae, J.-H.; Hyun, S.-S.; Seo, S.-Y.; Lee, Y.-S.; Lee, K.-O.; Kim, S.-H.; Lee, Y.-S.; Jeong, J. M.; Choi, N.-S.; Suh, Y.-G. Bioorg. Med. Chem. Lett. 2004, 14, 4519.

(15) Sasaki, S.; Kitamura, S.; Negoro, N.; Suzuki, M.; Tsujihata, Y.; Suzuki, N.; Santou, T.; Kanzaki, N.; Harada, M.; Tanaka, Y.; Kobayashi, M.; Tada, N.; Funami, M.; Tanaka, T.; Yamamoto, Y.; Fukatsu, K.; Yasuma, T.; Momose, Y. J. Med. Chem. 2011, 54, 1365.

(16) Patani, G. A.; LaVoie, E. J. Chem. Rev. 1996, 96, 3147.

(17) Lima, L. M.; Barreiro, E. J. Curr. Med. Chem. 2005, 12, 23.

(18) Zonta, C.; De Lucchi, O.; Volpicelli, R.; Cotarca, L. Top. Curr. Chem. 2007, 275, 131.

(19) Zheng, N.; McWilliams, J. C.; Fleitz, F. J.; Armstrong, J. D.; Volante, R. P. J. Org. Chem. 1998, 63, 9606.

(20) Giubellino, A.; Shi, Z.-D.; Jenkins, L. M.; Worthy, K. M.; Bindu, L. K.; Athauda, G. B.; Peruzzi, R.; Fisher, J.; Appella, E.; Burke, T. R.; Bottaro, D. P. J. Med. Chem. 2008, 51, 7459.

(21) Molander, G. A.; Yun, C.-S. Tetrahedron 2002, 58, 1465.

(22) Sharifi, A.; Abaee, M. S.; Mirzaei, M.; Naimi-Jamal, M. R. Asian J. Chem. 2010, 22, 6519.

(23) Procedure for Newman-Kwart Rearrangement: S-(2,5Dimethylnaphthalen-1-yl)dimethylcarbamothioate (12) A solution of $O$-(2,5-dimethylnaphthalen-1-yl)dimethylcarbamothioate $(\mathbf{1 1}, 300 \mathrm{mg}, 1.15 \mathrm{mmol})$ in $\mathrm{N}, \mathrm{N}$-diethylaniline (10 $\mathrm{mL}$ ) was equipped with sure-sealed round-bottom flask. And the solution was stirred for $12 \mathrm{~h}$ at $240{ }^{\circ} \mathrm{C}$. After cooling, the solution was quenched with $10 \% \mathrm{HCl}$ and diluted with EtOAc. The organic layer was washed with $\mathrm{H}_{2} \mathrm{O}$ and brine and dried with $\mathrm{MgSO}_{4}$. The filtered solution was concentrated, and the residue was purified with $\mathrm{SiO}_{2}$ column chromatography $($ EtOAc/hexane $=1: 10)$ to obtain the desired product as a yellowish oil (214 mg, 71\%). ${ }^{1} \mathrm{H}$ NMR $\left(600 \mathrm{MHz}, \mathrm{CDCl}_{3}\right): \delta=8.30$ $(\mathrm{d}, J=9.0 \mathrm{~Hz}, 1 \mathrm{H}), 8.01(\mathrm{~d}, J=9.0 \mathrm{~Hz}, 1 \mathrm{H}), 7.47(\mathrm{~d}, J=8.4 \mathrm{~Hz}$, $1 \mathrm{H}), 7.40(\mathrm{t}, J=7.2 \mathrm{~Hz}, 1 \mathrm{H}), 7.26(\mathrm{~d}, J=7.2 \mathrm{~Hz}, 1 \mathrm{H}), 3.27(\mathrm{~s}, 3 \mathrm{H})$, $3.01(\mathrm{~s}, 3 \mathrm{H}), 2.68(\mathrm{~s}, 3 \mathrm{H}), 2.64(\mathrm{~s}, 3 \mathrm{H}) \mathrm{ppm} .{ }^{13} \mathrm{C}$ NMR $(150 \mathrm{MHz}$, $\left.\mathrm{CDCl}_{3}\right): \delta=165.9,142.6,136.1,134.6,131.8,128.4,126.7,126.3$, 126.1, 124.9, 124.0, 22.5, 19.6 ppm. HRMS (ESI-TOF): $\mathrm{m} / z$ [M + $\mathrm{H}]^{+}$calcd for $\mathrm{C}_{15} \mathrm{H}_{18}$ NOS: 260.1104; found: 260.1103 . 
(24) Synthesis of Ethyl 2-[(2,5-dimethylnaphthalen-1-yl)thio]acetate (15)

To a solution of 2,5-dimethylnaphthalen-1-yl trifluoromethanesulfonate $(\mathbf{1 4}, 283 \mathrm{mg}, 0.930 \mathrm{mmol})$ in degassed toluene was added $\mathrm{Pd}_{2}(\mathrm{dba})_{3}(85 \mathrm{mg}, 0.093 \mathrm{mmol}), 1,1$ '-bis(diphenylphosphino)ferrocene ( $57 \mathrm{mg}, 0.102 \mathrm{mmol}$ ), NaOt-Bu (156 mg, 1.39 $\mathrm{mmol})$, and then ethyl thioglycolate $(97 \mu \mathrm{L}, 1.39 \mathrm{mmol})$ was added. And the reaction mixture was refluxed for 1 day. And then the reaction solvent was evaporated. The residue was purified with $\mathrm{SiO}_{2}$ column chromatography with EtOAc/hexane
(20:1) to obtain the desired product as a colorless oil (104 mg, 43\%). ${ }^{1} \mathrm{H}$ NMR $\left(600 \mathrm{MHz}, \mathrm{CDCl}_{3}\right): \delta=8.53(\mathrm{~d}, J=8.4 \mathrm{~Hz}, 1 \mathrm{H})$, $7.95(\mathrm{~d}, J=8.4 \mathrm{~Hz}, 1 \mathrm{H}), 7.46(\mathrm{t}, J=7.2 \mathrm{~Hz}, 1 \mathrm{H}), 7.43(\mathrm{~d}, J=9.0 \mathrm{~Hz}$, $1 \mathrm{H}), 7.30(\mathrm{~d}, J=7.2 \mathrm{~Hz}, 1 \mathrm{H}), 3.97(\mathrm{q}, J=7.2 \mathrm{~Hz}, 2 \mathrm{H}) 3.44(\mathrm{~s}, 2 \mathrm{H})$, $2.78(\mathrm{~s}, 3 \mathrm{H}), 2.69(\mathrm{~s}, 3 \mathrm{H}) \mathrm{ppm} .{ }^{13} \mathrm{C}$ NMR $\left(150 \mathrm{MHz}, \mathrm{CDCl}_{3}\right)$ : $\delta=169.9,141.9,135.4,134.8,132.0,129.1,128.4,126.7,126.2$, 125.7, 124.4, 61.2, 37.5, 22.3, 19.6, 13.9 ppm. HRMS (ESI-TOF): $\mathrm{m} / \mathrm{z}[\mathrm{M}+\mathrm{H}]^{+}$calcd for $\mathrm{C}_{15} \mathrm{H}_{17} \mathrm{O}_{2} \mathrm{~S}: 261.0944$; found: 261.0942 .

(25) Zimmer, H.; Lankin, D. C.; Horgan, S. W. Chem. Rev. 1971, 71, 229. 